\title{
Emergent Parsing and Generation with Generalized Chart
}

\author{
HASIDA Kôiti \\ Electrotechnical Laboratory \\ 1-1-4 Umezono, Tukuba, Ibaraki 305, Japan \\ E-mail: hasida@etl.go.jp
}

\begin{abstract}
A new, flexible inference method for Horn logic program is proposed. It is also a drastic generalization of chart parsing, partial instantiation of clauses in a program roughly corresponding to arcs in a chart. Chartlike parsing and semantic-head-driven generation emerge from this method. With a parsimonious instantiation scheme for ambiguity packing, the parsing complexity reduces to that of standard chart-based algorithms.
\end{abstract}

\section{Introduction}

Language use involves very complex interactions among very diverse types of information, not only syutactic one but also semantic, pragmatic, and so forth. It is hence inappropriate to assume any specific algorithm for syntactic parsing or generation, which prescribes particular processing directions (such as left-to-right, top-down and bottom-up) and is biased for specific: types of domain knowledge (such as a context-frec grammar). To account for the whole language use, we will have to put many such algorithms together, ending up with an intractably complicated model.

$\Lambda$ better strategy is to postulate no specific algorithms for parsing or generation or any particular task, but instead a single uniform computational method from which emerge various types of computation including parsing and generation depending upon various computational contexts.

For example, Earley decluction (Pereira \& Warmen, 1983) is a general procedure for dealing with Hor'n clauses which gives rise to Earley-like parsing when given a context-free grammar and a word string as the input. Shieber (1988) has generalized this method so as to adapt to sentence generation as well. Those methods fail to give rise to efficient computation for a wide varicty of contexts, however, because they prescribe processing directions such as left-to-right for parsing and bottom-up for generation. They also lack a general way of efficient ambiguity packing unlimited to context-free grammars. Hasida (1994a) proposes a more general inference method for clausal form logic programs which accounts for efficient parsing and generation as emergent phenomena. This method prescribes no fixed processing directions, and the way it packs ambiguity is not specific to context-frec grammars. However, it is rather complicated and has greater computational complexity than standard algorithms do.
In this paper we propose another inference method for Horn logie programs based on Masida (1994a), and show that efficient parsing and generation emerge from it. Like that of Hasida (1994a), this method is totally constraint-based in the sense that it presupposes no fixed directions of information flow, but it is more efficient owing to a parsimonions method of instantiation. In Section 2 we define this inference method, which is a generalization of chart parsing, and may be also thought of as a connection method or a sort of program transformation. Section 3 illustrates how efficient parsing and generation emerge from this method without any procedural stipulation specific to the task and the domain knowledge (syntactic constraints). Section 4 introduces a parsimonious instantiation metlod for ambiguity packing. We will show that owing to this methorl the efficiency reaches that of the standard algorithms with regard to context-free parsing. Section 5 concludes the paper by touching upon further research directions.

\section{Partial Instantiation}

A constraint is represented in terms of a Horn clanse progran such as below.

$$
\begin{aligned}
& \text { (a) }-p(A, B)-A=a(C) . \\
& \text { (b) } p(X, Y)-X=a(Y) . \\
& \text { (c) } p(U, W)-p(U, V)-p(V, W)
\end{aligned}
$$

Nanes beginning with capital letters represent variables, and the other nanes predicates and functors. The atomic formulat following the minus sign ate negative (body) literals, and the others are positive (head) literals. A clatse withont a positive literal is called a top clause, whose negation represents a goal (toplevel hypothesis), which corresponds to a query in Prolog. For instance, top clanse (a) in the above program is regarded as goal $\exists A, B, C\{p(A, B) \wedge A=a(C)\}$. In general, there may be several top elanses. The purpose of computation is to tell whether any goal is satisfiable, and if so obtain an answer substitution for the terms (variables) in a satisfiable goal. We consider the minimal Herbrand models as usmal. So the set of a1swer substitutions for $A$ in the above program is $\{a(B)$, $a(a(B)), a(a(a(B))), \cdots\}$.

A graphical representation of this program is shown in Figure 1. Here each clanse is the set of the literals enclosed in a clim closed curve. A link connecting agunents in a clatuse is the term (variable) filling in 


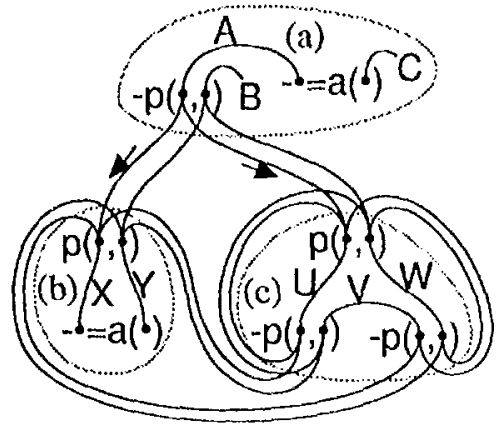

Figure 1: A graphical representation of a program.

those arguments. (It is a hyperlink when there are more than two arguments.) A transclausal link represents the unifiability between two corresponding argnments of two unifiable literals. (Neglect the arrows for a while.)

A hypothesis is a conjunction of atomic formulas and bindings. The premise of a clause (i.e., the conjunction of the atomic formulas and binclings which appear as negative literals) is a hypothesis. An expansion for a hypothesis is a way of combining (instances of) clauses by resolutions so as to translate the lypothesis to another hypothesis involving bindings only. We will refer to an expansion by the sequence of clanses in the order of leftmost application of resolution nsing their instances. ${ }^{1}$ In the above program, for example, expansion $\langle c, b, b\rangle$ translates the top-level lyypothesis $s(A, B) \wedge A=a(C)$ to a lypothesis $A=a(C)$ $\wedge C=a(B)$. An expansion of a clanse is an expansion of its premise. We will simply say 'an expansion' to mean an expansion of the top-level hypothesis. $\Lambda$ program represents a set of expansions, and the computation as discussed later is to transform it so as to figure out correct hypotheses while discarding the wrong expansions (those entailing wrong hypotheses).

We say that there is a dependency between two terms when those terms are nuified in some expansion, and the sequence of terms (including them) mediating this unification is called the dependency path of this dependency. In Figure 1, for instance, the dependency between $A$ and $X$ is mediated by dependency pall $A \cdot X$, $A \cdot U \cdot X, A \cdot U \cdot U \cdot X$, and so on. There is a dependency between $C$ and $B$, among other's, because of the miliat bility of the two $-\bullet=\mathrm{a}(\bullet) \mathrm{s}$, though this unifiability is not explicitly shown in Figure 1. We say a dependency between two terms is consistent when they are not bound by inconsistent bindings. All the dependencies in Figure 1 are consistent.

A solntion of the program is an expansion in which every dependency is consistent. So the computation we propose in this paper is to transform the given program in such a way that every dependency be consistent. To figure out dependencies, we use il symbolic operation called subsumption, and delete the parts of the program which contributes to wrong expansions

\footnotetext{
${ }^{1}$ Here we mention the order among the litemals in a clanse just for explanatory convenience. This order is not significint in the computation discussed later.
}

only. For example, suppose there is an inconsistent dependency between terms $\alpha$ and $\beta$. Wo create an instance $\beta^{\prime}$ of $\beta$ by subsumption operations to be discussed shortly, so that every expansion containing an instance of $\beta^{\prime}$ contains an instance of a dependency path between $\alpha$ and $\beta$. We can then delete the clause containing $\beta^{\prime}$ and probably some more parts of the program without affecting the declarative semantics of the program. Below we will clefine a computational procedure in such a way that the set of the possible expansions eventnally represent the set of all the solntions.

Subsumption operation is to create subsumption relationship. We regard each part (clause, atomic formula, term, atc.) of a program ats the sel of its instances, and say that a part $\xi$ of the program subsumes another part $\eta$ to mean that we explicitly know that $\xi \supseteq \eta$. Wo consider that a link is subsumed by $\delta$ if and only if one of the terms it links is subsumed by $\delta$. We say term $\delta$ is an origin of $\eta$ when $\eta$ is subsuned by $\delta$. In this paper we consirler that every origin is a bound term (the term filling in the first argument of a binding). Let us say that two clattses (or two literals) are equivalent when they are of the same form and for each pair of corresponding terms the two terms have the same set of origins.

Subsumption relation restricts the possibility of expansions so that if term $\eta$ is subsumed by another term $\delta$, then every expansion containing an instance of $\eta$ must also contain an instance of $\delta$. Subsunption relation is useful to encode structure sharing among expansions. In subsumption-based approaches, a term may subsume several non-unifiable terms and thus the first tern is shared among the latters. IIowever, that is impossible in mification-based approaches, where different expansions cannot share the same instance of a term or a clause.

A partially instantiated clause is a clatuse some of whose terms is subsumed by another term in possibly another clanse. For instance,

(1) $\mathrm{a}\left(\overline{\mathrm{A}_{i}}, \mathrm{Z}\right)-\mathrm{b}\left(\overline{\mathrm{A}_{i}}, \overline{\mathrm{A}_{j}}\right)-\mathrm{c}\left(\overline{\Lambda_{i}}, \mathrm{Z}\right)$.

is a partial instantiation of the following clanse:

(2) $a(X, Z)-b(X, Y)-c(Y, Z)$.

$\bar{A}$ represents a term sulsmmed by term $\wedge .{ }^{2}$ Ifereafter we saly just 'clause' to reler to both uninstintiated clatuses and partially instantiated clanses.

A program consisting of such clanses is a generalization of a clart (Kay, 1980). A chart is a graph whose nodes denote positions between words in a sentence and whose ares are regateled as context-fiee rules each instantiated partially with respect to at most two such positions. For instance, an active are from node $i$ to node $j$ labelled with $[A \rightarrow \bullet B \bullet C]$ is an instance of rule $A \rightarrow B C$ with both sides of $B$ instantiated by positions $i$ and $j$. 'lhis are apploximately corresponds to (1)..$^{3}$

\footnotetext{
2'This notation is problematic because it is nuclear whother two occurences of $\bar{A}$ in a claluse donote the same term. In this pelper they always do.

${ }^{3}$ Ilowever, an are in it chart does not precisely correspond to at partially instantiated clatse derived from is progran encoding
} 
A subsumption operation is to extend subsumption relation by possibly creating a partially instantiated clause, $A$ subsumption operation is characterized by the origin, the source, and the target. The origin (let it be $\delta$ ) is a bound term. The source $(\sigma)$ and the target $(\tau)$ are arguments. $\sigma$ should already be subsumed by the origin, but $\tau$ should not be so. They should be connected through a transclansal link $\lambda$. Let the literal containing $\sigma$ be $\rho$. Also let the literal containing $\tau$ be $\pi$, and the clause containing then be $\Phi$. There are two cases for subsumption, and in both cases $\sigma$ comes to be linked through $\lambda$ with an argument which is an instance of $\tau$ subsumed by $\delta$.

In the first case of subsumption operation, which we call unfolding, a partial instantiation $\Phi^{\prime}$ of $\Phi$ is created. They are equivalent except that the instance $\tau^{\prime}$ of $\tau$ in $\Phi^{\prime}$ is subsumed by $\delta$. After the unfolding, $\sigma$ is linked through $\lambda$ to the instance of $\tau$ in $\Phi^{\prime}$ instead of the original $\tau$, and accordingly $\rho$ is linked to the instance of $\pi$ in $\Phi^{\prime}$. Let $\tau^{\prime \prime}$ be $\tau$ after the unfolcling. Then $\tau^{\prime} \cup \tau^{\prime \prime}=\tau, \tau^{\prime} \cap \tau^{\prime \prime}=\emptyset$, and $\tau^{\prime}=\tau \cap \sigma$ hold. This implies $\tau^{\prime} \subseteq \delta$ and $\tau^{\prime \prime} \cap \sigma=\emptyset$. So $\tau^{\prime \prime}$ and $\sigma$ are not unifiable.

For instance, the two subsumption operations indicated by the two arrows in Figure 1 are unfoldings. In either case, the origin and the source are both $A$. The target in the left is $X$ and that in the right is $U$. We obtain the program in Figure 2 by these operations,

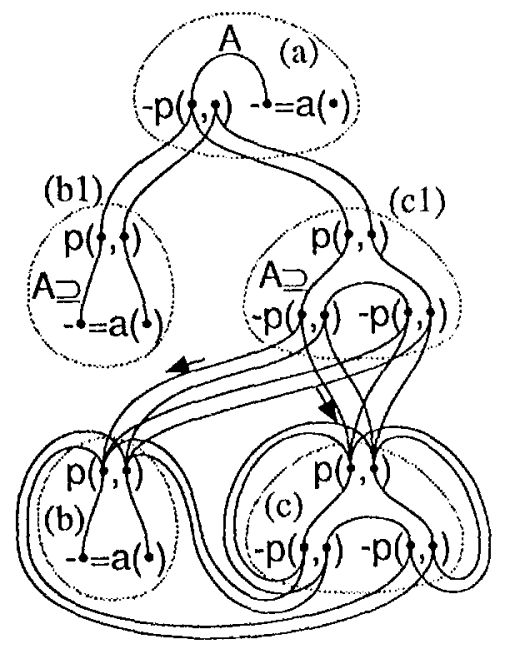

Figure 2: After snbsumptions to $X$ and $U$ by $A$.

where partial instantiation (bl) and (c1) of (b) and (c) have been created, respectively.

In Figure 1, the subsumption operation through the (invisible) link commecting $C$ and $Y$ is not exccutable now, because the unification represented by this link presupposes the unification of $A$ and $X$ through the dependency paths $A \cdot X, A \cdot U \cdot X, A \cdot U \cdot U \cdot X$, and so on. That is, it is only when $C$ subsumes an instance (let it be $Y^{\prime}$ ) of $Y$ that subsumption from $C$ to $Y^{\prime}$ is possible. (This subsumption is an unfolding without any copy,

a context-free grammar in a standard way. See Section 4 for further discussion. because then $C$ automatically subsumes $Y^{\prime}$.) Same for the subsumption in the opposite direction.

The second case of subsumption operation is called folding. It takes place when there is already a literal $\pi^{\prime}$ equivalent to $\pi$ except that its argument $r^{\prime}$ corresponding to $\tau$ is subsumed by $\delta$. In this case, no new instance of clause is created, but instead link $\lambda$ is switched so that it links $\sigma$ with $\tau^{\prime}$ and accordingly $\rho$ is linked with $\pi^{\prime}$. Iset $\tau^{\prime \prime}$ be $\tau$ after the unfolding. Then $\tau \cap \tau^{\prime}=\emptyset$ both before and after the folding, and $\sigma \cap \tau$ is subtracted from $\tau$ and added to $\tau^{\prime}$ by the folding. Folding is triggered when there exists literal $\pi^{\prime}$ as described above, and unfolding is executed otherwise. If there existed several such $\pi$ 's, folding takes place, creating as many instances of $\lambda$ and connecting to those $\pi^{\prime} \mathrm{s}$.

The two subsumption operations indicated in Figwre 2 are foldings. Actually, in the left, the $p(\bullet, \bullet)$ in (b1) and that in (b) are equivalent exeept that the first argument of the former is subsumed by $A$. So the link with the arrow and the parallel accompanying link are switched up to $p(\bullet, \bullet)$ in (b1). Similarly for the right subsumption. Shown in Figure 3 is the result.

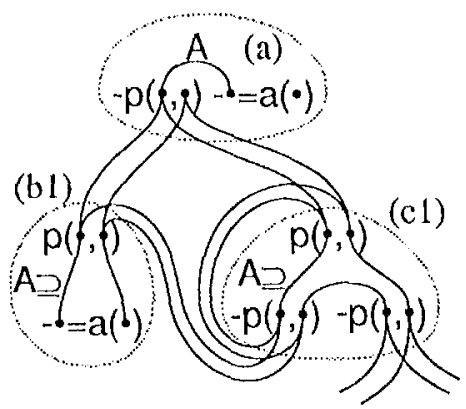

Figure 3: After foldings.

Note that the original program encodes a problem of partial parsing of a string beginning with " $a$ " Inder the context-free grammar consisting of the following rules.

$$
\begin{aligned}
& P \rightarrow a \\
& P \rightarrow P P
\end{aligned}
$$

'The result in Figure 3 encoles the infinitely many possible parses of this incomplete sentence. Note also that lere the subsumption from $C$ to the instance of $Y$ in (b1) would be possible if $C$ were bound. The next section contains relevant examples.

When a link is subsumed by two terms bound by two inconsistent bindings ( that link is deleted, surrounding clauses possibly being deleted if some of their atomic formulas are linked with no atomic formula any more.

For the sake of simplicity, we mainly consider inputbound programs in this paper. We say a program is input-bound when every depenclency path between bound terms comnects a term in a top clause and one in a non-top clanse. The progran in Figure 1 and the ones for parsing and generation in the following section are all input-bound programs. For input-bound 
programs, we have only to consider subsumptions by terms in top clauses: input-driven computation. Also, in input-driven computation for input-bound programs we do not lave to worry about duplications of origins by subsumptions.

Both subsumption and deletion preserve the declarative semantics of the program (the set of the solutions), though we skip a detailed proof due to the spatce limitation. So when they are not applicable any more, every expansion is a solution and vice versa. For inputbound programs, the input-driven computation always terminates within time polynomial as to the size of the program. This is because there are at most $n^{m}$ partially instantiated clauses derived from a clause with $m$ terms, where $n$ is the size of the input (the number of bound terms in the top clause(s)), and accordingly there are polynomially many transclausal links. Obviously, partially instantiated clanses and new transclausal links are each created in constant time. It is also clear that each folding terminates in polynomial time.

\section{Parsing and Generation}

Here we show that chart-like parsing and semantichead-driven generation emerge from the above complntational method. We discuss examples of parsing ancl generation both on the basis of the following grammat.

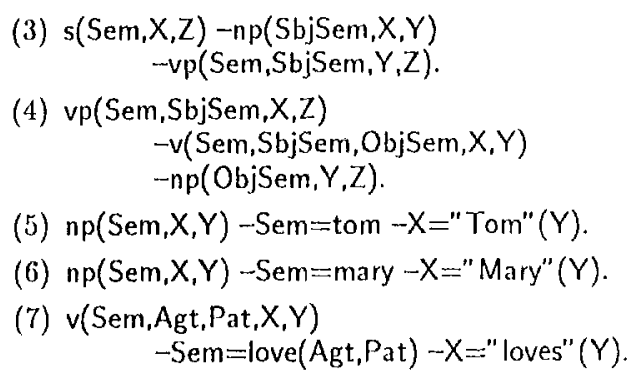

Since we have already mentioned ambiguity packing in the previous section, bolow we do not explicitly deal with ambiguity but instead discuss just one sentence structure in both parsing and generalion.

Iet us first consider parsing of sentence "Tom loves Mary'. The problem is encoled by the progrium in Figure 4. The input-driven computation procecess as shown by the arrows, which represent subsumption operations taking place in the ordering indicated by the labelling numbers. $\Lambda$ thick dependency path is processed by successive subsumptions with the same origin. The only subsumption operations executable in the initial situation is the one numbered 1 and after that the one numbered 2 , along the thick path between $A_{0}$ and $X$ in (5). As the result of these unfoldings, we obtain the following clauses.

$$
\begin{aligned}
& \text { (8) } s\left(\operatorname{Sem}, \overline{A_{0}}, Z\right)-n p\left(\operatorname{Sbj} \operatorname{Sem}, \widetilde{A_{0}}, Y\right) \\
& -v p(\text { Sem,SbjSem, } Y, Z) \text {. } \\
& \text { (9) } \mathrm{np}\left(\operatorname{Sem}, \overline{A_{0}}, \overline{A_{1}}\right)-\operatorname{Sem}=\text { tom }-\overline{A_{0}}=\text { "Tom" }\left(\overline{A_{1}}\right) \text {. }
\end{aligned}
$$

Of course other partially instantiated clauses may be created here from definition clauses of $s$ other than (3) and those of np other than (5), but we onit them liere and concentrate on just one solution.

Now the copy of link with the arrow numbered 3 connected to (9) can mediate subsumption operations. So the subsumption operation indicated that arrow is triggered, though that does not duplicate (9) because $A_{1}$ already subsumes the target. The result is alrealy reflected in (9). The subsequent subsumption operations numbered 4,5 , and 6 will yield the following clauses.

$$
\begin{aligned}
& \text { (1.0) } s\left(\operatorname{Sem}, \widehat{A_{0}}, Z\right)-n p\left(\operatorname{Sbj} \operatorname{Sem}, \overline{A_{0}}, \overline{A_{1}}\right) \\
& -v p\left(\operatorname{Sem}, \operatorname{Sbj} \operatorname{Sem}, \overline{A_{1}}, Z\right) \text {. } \\
& \text { (11) } v p\left(\operatorname{Sem}, \operatorname{SbjSem}, \overline{A_{1}}, Z\right) \\
& -v\left(\text { Sem,SbjSem,ObjSem, } \overline{A_{1}}, Y\right) \\
& -n p(O b j S e m, Y, Z) \text {. } \\
& \text { (12) } v\left(\text { Sem, Agt, Pat }, \widehat{A_{1}}, \overline{A_{2}}\right)-\operatorname{Sem}=\text { love (Agt,Pat) } \\
& -\overline{A_{1}}=\text { "loves" }\left(\overline{A_{2}}\right) \text {. }
\end{aligned}
$$

Now the subsunption operations by $A_{2}$ are commenced, che to the creation of (12). Accordingly, the following clanses are croated, and the parsing is finished.

$$
\begin{aligned}
& \text { (13) } s\left(\operatorname{Sem}, \overline{A_{0}}, \overline{A_{3}}\right)-n p\left(\operatorname{SbjSem}, \overline{A_{0}}, \overline{A_{1}}\right) \\
& -v p\left(\operatorname{Sem}, \operatorname{SbjSem}, \overline{A_{1}}, \overline{A_{3}}\right) \text {. } \\
& \text { (14) } v p\left(\text { Sem, SbjSem, } \overline{A_{1}}, \overline{A_{3}}\right) \\
& -v\left(\text { Sem,SbjSem,ObjSem, } \overline{A_{1}}, \overline{A_{2}}\right) \\
& -n p\left(O b j S e m, \overline{A_{2}}, \overline{A_{3}}\right) \text {. } \\
& \text { (15) } n p\left(\operatorname{Sem}, \overline{A_{2}}, \overline{A_{3}}\right)-\operatorname{Sem}=\operatorname{mary}-\overline{A_{2}}=" \operatorname{Mary} "\left(\overline{A_{3}}\right) \text {. }
\end{aligned}
$$

From the earlier discussion, in the case of contexttree parsing the number of clauses created there is $O\left(n^{M}\right)$, where $n$ is the number of the input words ancl $M$ the maximmm number of the occurences of non-terminal symbols in a context-free rule. This is larger than the space complexity of the standard parsing algorithms, but later we will show how to improve the efficiency so as to be equivalent to the standard algoritluns.

No particular order anong the subsumption operations is prescribed in the above computation, and so it is not inherently linited to top-down or bottom11p. Note also that the left-to-right processing order anong the input words is derived from the definition strong link, rather than stipulated as in Earley deduction, annong others. Wo can aecount for ishand-driven parsing as well, by allowing links between bindings to trigger subsunptions more earlier.

Let us next take a look at sentence generation. Consider the progrimn shown in Figure 5. IIne the input is senantic structure love(tom, mary). Again the computational process is indicated by the numbered arrows. $\sigma^{\prime}$ takes place after 5 , but the order anong 6,7 , and $6^{\prime}$ is arbitary as long as 6 should be before 7. So the only possible sulsumption operation in the beginning is the ones by Love, which go throngh the thick curve comnecting Love and the $X$ in (4). This creates the following elanse, among others.

(16) $v(\overrightarrow{\text { Love }}, \overline{\mathrm{Tom}}, \overline{\mathrm{M}}$ ry $, X, Y)$

$$
-\overline{\text { Love }}=\text { love }(\overline{\text { Tom }}, \overline{\text { Mary }})-X=" \text { loves" }(Y) \text {. }
$$




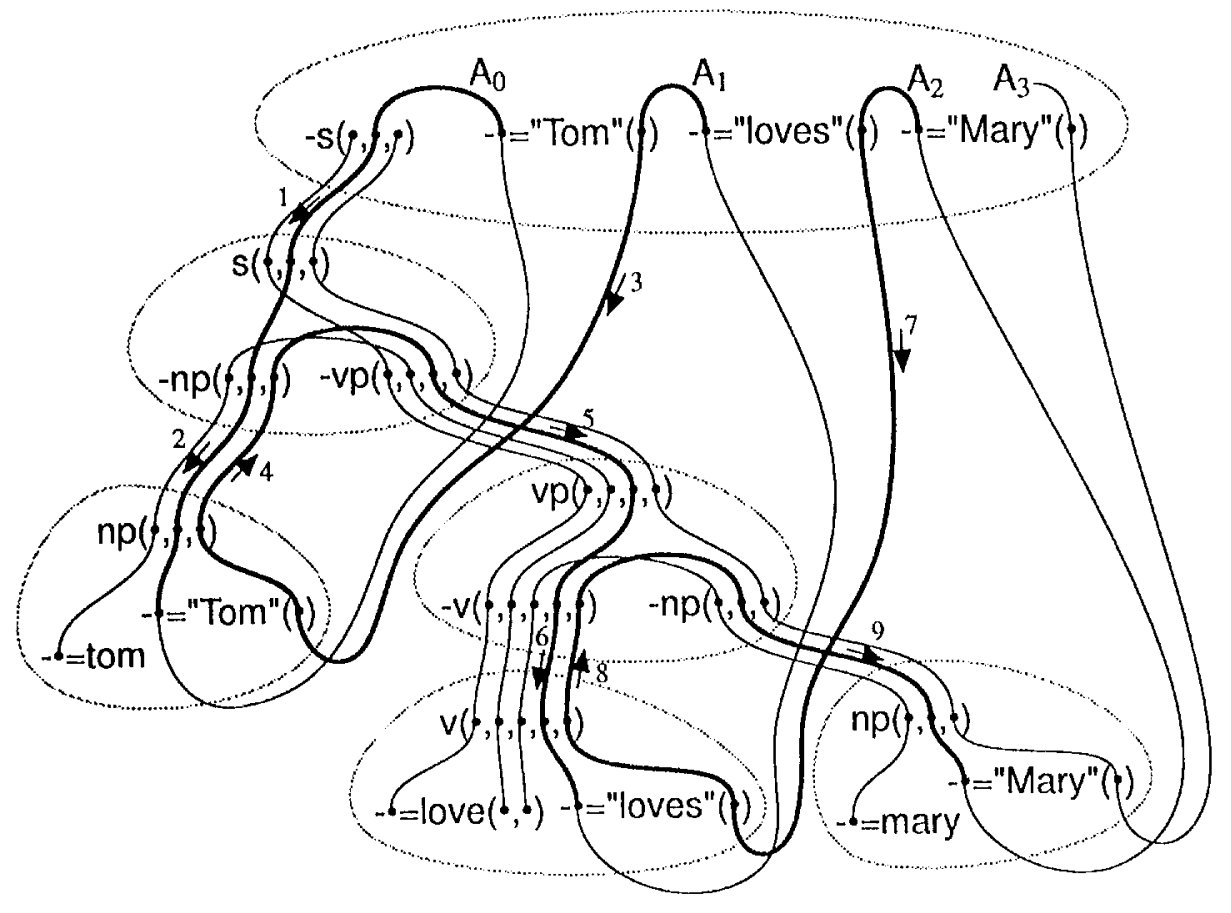

Figure 4: Parsing

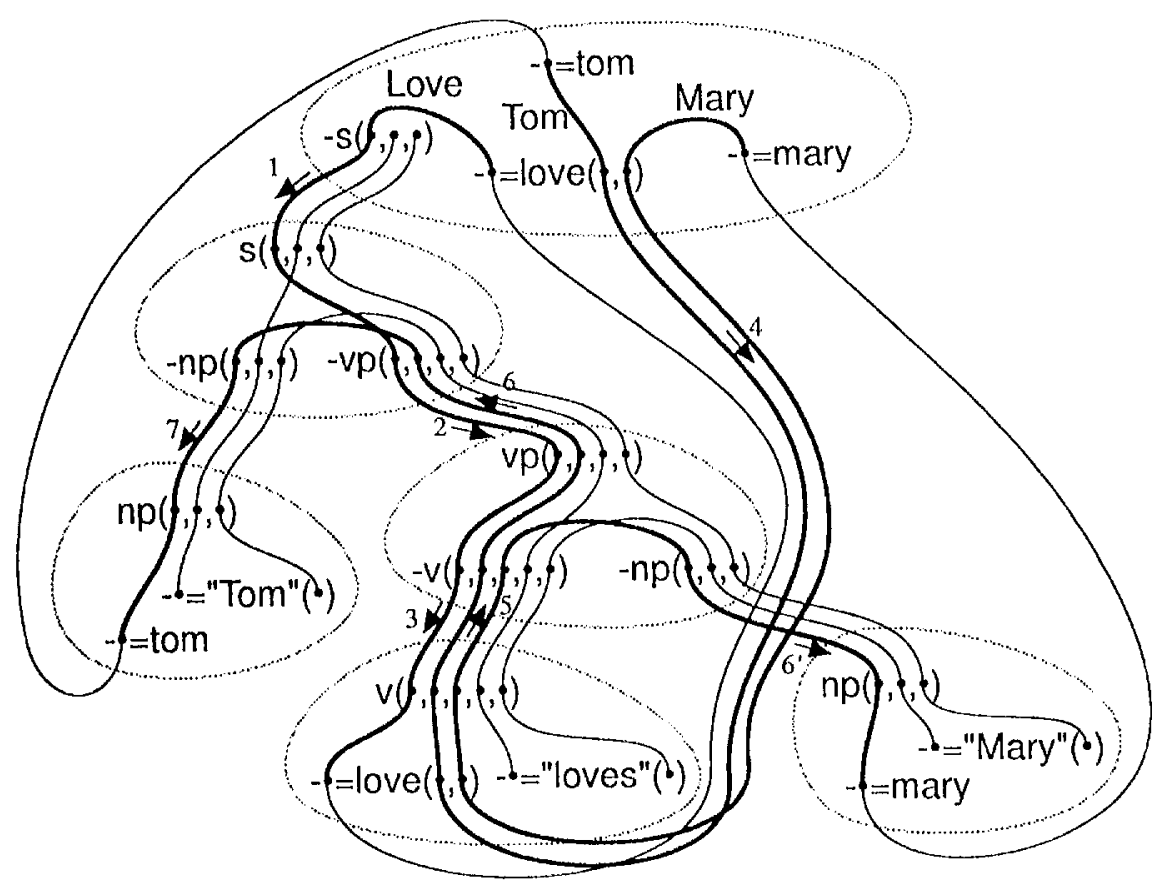

Figuie 5: Generation 
Now subsumption operations can go through the copies of the other two thick curves. So we are creating the following clanses, among others.

(17) $s(\overline{\text { Love }}, X, Z)-n p(\widehat{\text { Tom }}, X, Y)-v p(\overline{\text { Love }}, \overline{\text { Tom }}, Y, Z)$.

(18) $v p(\overline{\text { Love, Tom }}, X, Z)-v(\overrightarrow{\text { Love, }} \overline{\text { Tom }}, \overline{\text { Mary }}, X, Y)$ $-n p(\overline{\text { Mary, }} Y, Z)$.

(19) $n p(\overline{T o m}, X, Y)-\overline{T o m}=$ tom $-X="$ Tom" $(Y)$.

(20) $n p(\overline{\text { Mary }}, X, Y)-\overline{\text { Mary }}=$ mary $-X=$ "Mary" $(Y)$.

Note that this generation process amounts to a generalization of semantic-head-driven generation (Shieber, van Noord, \& Moore, 1989). The order among the retrievals of semantic heads is the oreler of subsumption operations by different terms in the input sentantic structure, just as with the processing order among words in the case of parsing. ${ }^{4}$ lso as in the case of parsing, the computational complexity of such a generation is polynomial with respect to the size of the input semantic structure, provided that the progran is input-bound and the computation is input-driven. Althongh the above example deals with only a single sentence structure, in general cases ambiguity pakking naturally takes place just as with parsing of anbignous sentences.

Under the restriction that the program be inputbound, the grammar cannot employ feature structures prevalent in the current linguistic theories, and also nuust be semantically monotonic (Shieber ot al., 1989) ${ }^{5}$ The proposed method can be generalized so ats to romove this restriction, though the details do not lit in the allowed space. This generalization makes it possible to deal with feature structures and semantically non-monotonic grammars. Of course the computation is not any more generally guaranteed to terminate (because Horn programs can encode Thring machines), but our method still has a better termination property than more simplistic ones such as Prolog interpreter or Barley deduction. For instance, endless expansion of left recursion or SUBCAT list, which would happen in simple top-down computations, is avoided owing to folding.

\section{Incremental Copy}

The parsing process discussed aloove is computationally more complex than chart parsing. Here we inprove our method by introducing a more efficient schent for ambiguity packing and thins reduce the parsing complexity to that of chart parsing, which is $O\left(n^{2}\right)$ for space and $O\left(n^{3}\right)$ for time.

The present inefficiency is due to excessive multiplication of clauses: much more partially instantiated clauses are created than ares in a clart. So let us suppose that a subsumption operation does not duplicate a whole clause but only some part of it, so that a clause is copied incrementally, as shown in Figne 6. Wo assume that a subsumption to an argument of a

\footnotetext{
"So the semantic-load-driven generation parallels better with left-to-right parsing than with syntactic-head-driven parsing.

${ }^{5}$ The semantic monotonicity is practically same ats the input boundness with regard to semantic structures.
}

literal copies the term filling in that argument, the literal, and some other literals which mention that term, muless there have already been the terms and literals to be thus created. Subscript $i$ of a literal indicates that it is created by the $i$-th subsumption operation.

We must ensure that this partial copying be semantically equivalent to the copying of whole clatuses. That is a trivial business when there are just one or two literals in the original clause. The case where there are more than three literals recluces to the case where there are exactly three literals, by grouping several literals; connected directly (through terms) and treat them as if they were one literal. So below let us consider the case where there are three literals in a clause.

$\Lambda$ non-trivial check must be done in such it case as in the lower right of Figure 6. Here you must copy $-r(\bullet, \bullet)_{2}$ and $-q(\bullet, \bullet)_{1}$ but not $-q(\bullet, \bullet)$, becatuse $-r(\bullet, \bullet)_{2}$ is compatible with $-q(\bullet, \bullet)_{1}$ but not with $-q(\bullet, \bullet)$. We say that a set of literals are compatible when there is an instance of the clanse which involves an instance of each of those literals. Also, two literals are said to be heterogeneous when they have different originals in the original minstantiated clanse. (The original of an original literal is itself.) In general, when a sulbsumption operation copies two heterogeneons, directly connected literals and creates two directly connected literals, the necessary and sufficient condition for this partial copy to be semantically equivalent to the fullchatuse copy is oloviously that the former two literals be compatible.

When two of the original literals are not comnected directly with each other, two heterogeneous literals which have directly connected originals are compatible iff they are also directly connected; we need not. consider two literals whose originals are not directly connected, because one subsumption operation does not copy stech literals at a time. When all of the three original literals are connected clirectly with each other, two heterogeneous literals are compatible ifl they are connected not only directly but also through another literal heterogeneous to both. In fact, $-r(\bullet, \bullet)_{2}$ and $-q(\bullet, \bullet)_{1}$ are comnected both through term $\xi$ and through $p(\bullet, \bullet)_{2}$, but $-r(\bullet, \bullet)_{2}$ and $-q(\bullet, \bullet)$ are not contnected through any instance of the original $p(\bullet, \bullet)$.

In the case of context-free parsing $O\left(n^{2}\right)$ literals are created, where $n$ is the number of words in the input, string, provided that the origins of sulsumptions are the positions between the input words only, rlue to the input-driven eomputation. Since there are just a constant times more links than literals, the space complexity of context-free parsing hence becomes $O\left(n^{2}\right)$ in our method. The time complexity is $O\left(n^{3}\right)$, because there are $O(n)$ different ways of making each literal. Now the correspondence with chart parsing is more $\mathrm{ex}$ act. $A$ are in the chatt corresponds to an instintiaterl literal. For instance, are $[A \rightarrow \bullet B \bullet C]$ from node $i$ to node $j$ corresponds to iustantiated literal $-b\left(\overline{A_{i}}, \overline{A_{j}}\right)$, and $[A \rightarrow \bullet B C \bullet \mid$ lrom node $i$ to node $j$ corresponds to a $\left(\overline{A_{i}}, \overline{A_{j}}\right)$. For a context-frec rule with more than two symbols in the right-hand side, we can gronp several literals to one as mentioned above and reduce it to a rule with just two symbols in the right-hand side. 

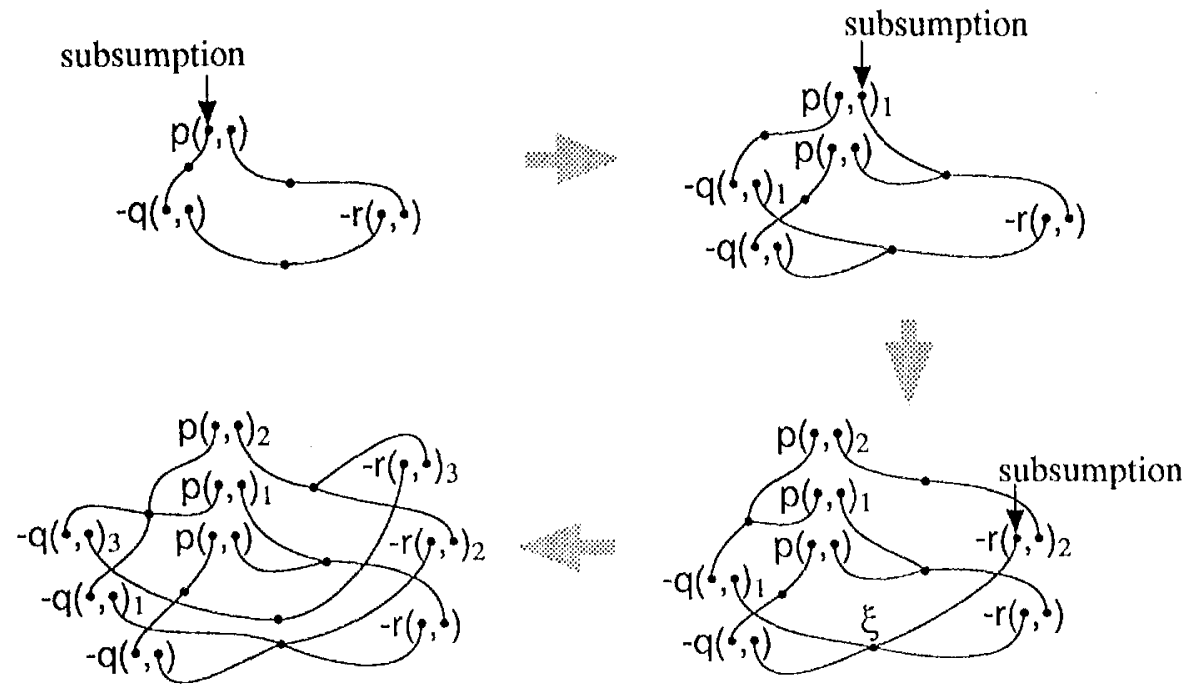

Figure 6: Sulsumptions with Incremental Copy

\section{Concluding Remarks}

We have proposed a flexible inference method for Horn logic programs. The computation bised on it is a sort of program transformation, and chart parsing and semantic-head-driven generation are epiphenomena emergent thereof. The proposed method has nothing specific to parsing, generation, context-frec grammar, or the like. This indicates that there is no need for any special algorithms of parsing or generation, or perhaps iny other aspect of natural language processing.

The idea reported above has alceady been partially implemented and applied to spoken langruage understanding (Nagao, Hasida, \& Miyata, 1993), and an account of how the roles of speaker and hearer may switch in the midst of a sentence (Fasida, Nagao, \& Miyata, 1993). Although this line of work has incorporated a notion of dymamics (Iasida, 1994b) as the declarative semantics to control context-sensitive computation, we are planning to replace dynamics with probability. For input-bound programs together with input-driven computation, it is quite straightforward to define probabilistic semantices as a natural extension of stochastic context-free grannars, among others, because all the body literals are probabilistically independent in that case. We would like to report soon on a general treatment of probabilistically dependent literals while preserving the efficient structure sharing, which will guarantec efficient computation and learning.

\section{Reference}

Hasida, K., Nagao, K., \& Miyatia, T. (1993). Joint Utterance: Intrasentential Speaker/Hearer Switch as an Emergent Phenomenon. In $\mathrm{Ba}$ jcsy, R. (Ed.), Proceedlings of the 13th International Joint Conference on Avtificial Intelligenct. Chambéry.
Ilasicka, K. (1994a). Common Henristics for Parsing, Generation, and Whatever .... In Straalkowski, 'T. (Fid.), Reversible Grammar in Natural Language Processing. Kluwer Aculenic: Publisher, Dordrecht.

Hasida, K. (1994b). Dynamics of Symbol Systems. Now Generation Computing, 12(3). to appear in May 1994.

Kay, M. (1980). Algorithm Schemata and Datid Structures in Syntactic Processing. Tech. rep., XERoX Palo Alto Research Center, Palo Alto, California.

Nagao, K., Iasiclat, K., \& Miyata, 'T. (1993). Understancling Spoken Natural Language with OmniDirectional Information Flow. In Procedings of the 13th International Joint Conference on Arlificind Intelligences.

Pereira, F. C. N., \& Warren, D. IT. D. (1983). Parsing as Deduction. In Proceedings of the 21st Annual Mecting of $A C L$, pp. 137144.

Shicber, S. M. (1988). A Uniform Architecture for Parsing and Generation. In Procedlings of the 12th International Conference on Computational linguistics, pp. 614-619.

Shieber, S. M., van Noord, G., \& Moore, R. C. (1989). A Semantic-Fead-Driven Gencration Algorithun for Unification-Based Formalisms. In Procedings of the 27th Annual Meating of the Associntion for Computational Lingatstics, pp. 7-17. 